\title{
Impact of Maternal Intrapartum Antibiotics, and Caesarean Section with and without Labour on Bifidobacterium and Other Infant Gut Microbiota
}

\author{
Yuan Yao Chen ${ }^{1}$, Xin Zhao ${ }^{1}$, Wolfgang Moeder ${ }^{2}$, Hein M. Tun ${ }^{1,3}{ }^{\circledR}$, Elinor Simons ${ }^{4}$, Piushkumar J. Mandhane ${ }^{1}$, \\ Theo J. Moraes ${ }^{5}$, , Stuart E. Turvey ${ }^{6}$, Padmaja Subbarao ${ }^{5}$, James A. Scott ${ }^{2} \mathbb{D}$ and Anita L. Kozyrskyj ${ }^{1, *}$ \\ 1 Department of Pediatrics, Faculty of Medicine and Dentistry, University of Alberta, \\ Edmonton, AB T6G 1C9, Canada; yuanyao@ualberta.ca (Y.Y.C.); xzhao1@ualberta.ca (X.Z.); \\ heinmtun@hku.hk (H.M.T.); mandhane@ualberta.ca (P.J.M.) \\ 2 Dalla Lana School of Public Health, University of Toronto, Toronto, ON M5T 1R4, Canada; \\ wolfgang.moeder@utoronto.ca (W.M.); james.scott@utoronto.ca (J.A.S.) \\ 3 HKU-Pasteur Research Pole, School of Public Health, University of Hong Kong, \\ Hong Kong SAR 999077, China \\ 4 Department of Pediatrics and Child Health, Children's Hospital Research Institute of Manitoba, University of \\ Manitoba, Winnipeg, MB R3E 0J9, Canada; Elinor.Simons@umanitoba.ca \\ 5 Department of Pediatrics and Physiology, Hospital for Sick Children, University of Toronto, \\ Toronto, ON M5G 1X8, Canada; theo.moraes@sickkids.ca (T.J.M.); padmaja.subbarao@sickkids.ca (P.S.) \\ check for \\ updates \\ Citation: Chen, Y.Y.; Zhao, X.; \\ 6 Department of Pediatrics, Child and Family Research Institute, BC Children's Hospital, University of British \\ Columbia, Vancouver, BC V5Z 4H4, Canada; sturvey@bcchr.ca \\ * Correspondence: Anita Kozyrskyj kozyrsky@ualberta.ca
} Moeder, W.; Tun, H.M.; Simons, E.; Mandhane, P.J.; Moraes, T.J.; Turvey, S.E.; Subbarao, P.; Scott, J.A.; et al. Impact of Maternal Intrapartum Antibiotics, and Caesarean Section with and without Labour on Bifidobacterium and Other Infant Gut Microbiota. Microorganisms 2021, 9 , 1847. https://doi.org/10.3390/ microorganisms 9091847

Academic Editors: Gonzalo Solís Sánchez and Miguel Gueimonde

Received: 14 July 2021

Accepted: 25 August 2021

Published: 31 August 2021

Publisher's Note: MDPI stays neutral with regard to jurisdictional claims in published maps and institutional affiliations.

Copyright: (C) 2021 by the authors. Licensee MDPI, Basel, Switzerland. This article is an open access article distributed under the terms and conditions of the Creative Commons Attribution (CC BY) license (https:// creativecommons.org/licenses/by/ $4.0 /)$.

Abstract: Background and Aims: Few studies consider the joint effect of multiple factors related to birth, delivery mode, intrapartum antibiotic prophylaxis and the onset of labour, on the abundance of Bifidobacterium and the quantity of this genus and its species Bifidobacterium longum subsp. infantis in the infant gut microbiota. We implemented such a study. Methods: Among 1654 Canadian fullterm infants, the gut microbiota of faecal samples collected at 3 months were profiled by $16 \mathrm{~S}$ rRNA sequencing; the genus Bifidobacterium and Bifidobacterium longum subsp. infantis were quantified by qPCR. Associations between Bifidobacterium and other gut microbiota were examined by Spearman's rank correlation. Results: Following vaginal birth, maternal IAP exposure was associated with reduced absolute quantities of bifidobacteria among vaginally delivered infants (6.80 vs. $7.14 \log _{10}$ (gene-copies/g faeces), $p<0.05$ ), as well as their lowered abundance relative to other gut microbiota. IAP differences in infant gut bifidobacterial quantity were independent of maternal pre-pregnancy body-mass-index (BMI), and remarkably, they were limited to breastfed infants. Pre-pregnancy BMI adjustment revealed negative associations between absolute quantities of bifidobacteria and CS with or without labour in non-breastfed infants, and CS with labour in exclusively breastfed infants. Significant correlations between Bifidobacterium abundance and other microbial taxa were observed. Conclusions: This study documented the impact of the birth mode and feeding status on the abundance of gut Bifidobacterium, and pointed to the important ecological role of the genus Bifidobacterium in gut microbiota due to its strong interaction with other gut microbiota in early infancy.

Keywords: Bifidobacterium; Bifidobacterium longum subsp. infantis; infant gut microbiota; caesarean section; maternal intrapartum antibiotics; labour; breastfeeding

\section{Introduction}

How we are born shapes the development of our gut microbiota. Caesarean section (CS) birth alters the gut microbiota of the young infant with its signature influence being the substantial reduction in the abundance of Bacteroidetes species [1,2]. Bifidobacteria are also reportedly reduced in number with CS vs. vaginal delivery, as early as 3-5 days after caesarean birth [3], but these differences are less apparent 6 months later [2,4]. Mother's 
milk, specifically its constituent human milk oligosaccharides (HMOs), was credited with an expansion of bifidobacteria [5]. The original source of bifidobacterial species depletion in infancy was attributed to surgical interference of maternal microbiota transfer during birth [6,7]. Interestingly, bifidobacteria are minimally depleted immediately after birth in the meconium (first stool) of infants born by caesarean, with or without labour, compared to vaginal delivery $[8,9]$.

Whereas the imprint of caesarean section is substantive, other birth events also influence the infant gut microbial composition during the first year of life. Although not often reported, prolonged labour during birth is associated with reduced abundance of Bifidobacterium [10]. As vaginal microbes are considered one of the primary sources of "seeding" or transferring of microorganisms from mother to newborn $[6,11]$, comparing the microbiota between infants delivered by CS with and without labour is worthy of study. In addition, newborns are exposed to maternal intrapartum antibiotic prophylaxis (IAP) according to clinical practice guidelines for the prevention of maternal infection associated with CS delivery or prophylaxis of vaginal Group B Streptococcus (GBS) in North America [12]. Within 2 days of IAP, newborn gut microbiota are depleted in bifidobacteria [13]. However, the impact of maternal IAP exposure on the abundance of Bifidobacterium at 3-4 months was not found in a subpopulation of the CHILD birth cohort [14]. In this study and others, whole microbial community profiling of infant faecal samples was conducted by $16 \mathrm{~S}$ sequencing, in which universal primers under-represent the presence of bifidobacteria $[15,16]$.

Members of the genus Bifidobacterium are pioneer gut colonizers and are considered foundational microbiota members that influence the early-life gut microbial community and exert positive effects on host health [17]. In addition to birth, infant diet shapes the gut microbiota of early life, with the greatest difference between breast-fed and formula-fed infants being the abundance of Bifidobacterium [18,19]. Breast milk is a rich source of glycans, dominated by HMOs, which selectively encourages the growth of Bifidobacterium $[5,20]$. Indeed, formula-feeding, which leads to a deficiency of Bifidobacterium, is associated with detrimental health outcomes, such as overweight, allergies and asthma [21,22].

Although the influence of breastfeeding in promoting Bifidobacterium in gut microbiota is well-known, factors related to birth, such as maternal IAP exposure and CS with labour, have been less studied. The objective of our paper was to fill this gap in the literature and build on findings from our previous research [14] to determine the association between maternal IAP, CS with and without labour, and genus Bifidobacterium and other gut microbial taxa in early infancy. Faeces were collected from infants at 3 months of age, and profiled by $16 S$ rRNA sequencing, as well as quantitative PCR (qPCR), to provide an accurate measure of the absolute quantities of bifidobacteria. Breastfeeding status was taken into account.

\section{Methods}

\subsection{Study Design}

This study includes a subset of 1654 infants enrolled in the Canadian Healthy Infant Longitudinal Development (CHILD) Study between 2009 and 2012 (www.childstudy.ca, accessed on 1 May 2021), in which 3-month infant faecal samples were additionally profiled for Bifidobacterium by qPCR. Mothers of studied infants were recruited in their second or third trimester of pregnancy and their newborns enrolled if they were a singleton live birth at 35 weeks of gestation with a birth weight of $2500 \mathrm{~g}$. In vitro fertilized births were excluded to avoid multiple gestation or preterm births. Hospital birth records provided information on sex, mode of delivery (vaginal, elective and emergency caesarean), duration of the first and second stage of labour, and maternal IAP. Women colonized with vaginal GBS or delivered by caesarean section received intrapartum administration of penicillin and cefazolin, respectively. These data were utilized to classify infants into mutually exclusive groups as follows: vaginal delivery with no IAP, vaginal delivery with IAP, CS delivery with no labour and CS delivery with labour. CS delivery was also defined clinically as elective CS (also known as scheduled or planned or without labour) and emergency CS, 
as per our previous study [14]. Although emergency CS delivery is commonly performed after the onset of labour [23], some emergency caesareans are performed before labour due to fetal distress. Data on maternal characteristics, including ethnicity, age, education level and prenatal smoking, as well as the infant characteristics, such as gestational age, breastfeeding status and infant antibiotic use before 3 months, were obtained from standardized questionnaires completed by mothers. Maternal pre-pregnancy body-mass-index (BMI) and prepregnancy weight status was measured as previously described [24]. All infants provided faecal samples at 3 months, which were analyzed by $16 \mathrm{~s}$ rRNA sequencing and targeted qPCR to detect total bifidobacteria and Bifidobacterium longum subsp. infantis. A directed acyclic graph (DAG) was generated to illustrate putative relations between the birth mode and infant gut microbiota at 3 months using the daggity.net program (Accessed date: 01 May 2021) [25], which allows the selection of the minimum set of covariates required for adjustment $[26,27]$ (Figure S1). Potential confounding factors selected by DAG (pre-pregnancy BMI, maternal ethnicity and education) were adjusted in the multivariable regression models if they caused a 15\% change in the beta-coefficient for birth mode. As infant antibiotic treatment can be a confounding factor or a mediator (as shown in the DAG), we also tested this variable in models with the $15 \%$ estimate rule. Only maternal prepregnancy BMI met the $15 \%$ criterion and was adjusted in regression models. This microbiome study of the CHILD cohort was approved by the Human Research Ethics Boards of the University of Alberta (Pro00010073; 1 November 2020).

\subsection{Faecal Microbiota Analysis}

Sample collection, DNA isolation and amplification, 16S rRNA sequencing and taxonomic classification were performed as previously described $[14,28]$. Briefly, faecal samples of 5-10 g, collected from infant diapers during home-visits conducted at 3-4 months of age by a research assistant or parents according to an approved protocol, were refrigerated after collection, aliquoted and stored at $-80{ }^{\circ} \mathrm{C}$ until analysis. Genomic DNA was isolated from frozen stool samples (80 to $200 \mathrm{mg}$ ) using the QIAamp DNA Stool Mini kit (Qiagen, Venlo, the Netherlands) according to the manufacturer's instructions. The V4 hypervariable region of the bacterial 16S rRNA gene was amplified by PCR with universal bacterial primers: V4-515f: 5'-AAT GAT ACG GCG ACC ACC GAG ATC TAC ACT ATG GTA ATT GTG TGC CAG CMG CCG CGG TAA-3', V4-806r: 5'-CAA GCA GAA GAC GGC ATA CGA GAT XXXXXXXXXXXX AGT CAG TCA GCC GGA CTA CHV GGG TWT CTA AT-3'. PCR products were paired-end sequenced on an Illumina MiSeq platform. Following a QIIME pipeline (v1.6.0, qiime.org, accessed on 1 May 2021) [29], forward and reverse reads were assembled, demultiplexed and filtered against the GreenGenes bacterial reference database (v13.8) [30] to discard the sequences with less than 60\% similarity. Resultant sequences were clustered using the closed-picking algorithm at $97 \%$ similarity by USEARCH, and taxonomic assignment was carried out using the RDP classifier [31]. Finally, microbiota data were rarefied to 13,000 sequences per sample and relative abundances were calculated.

\subsection{Quantitative PCR ( $q P C R$ ) for Total Bifidobacteria and Bifidobacterium longum subsp. Infantis Detection}

A quantitative PCR assay of DNA isolated from infant faecal samples was conducted following a published protocol [32]. To minimize differential inhibitory effects due to variable concentrations of genomic template DNA in qPCR, all template DNA samples were first normalized by dilution to $1 \mathrm{ng} / \mu \mathrm{L}$. Then, each multiplex assay was prepared to contain the 1X QuantiNova Multiplex PCR Kit (Qiagen, Venlo, the Netherlands), $0.4 \mu \mathrm{M}$ of each primer, $0.25 \mu \mathrm{M}$ of each probe and $1 \mu \mathrm{L}(1 \mathrm{ng} / \mu \mathrm{L})$ of sample DNA in a final volume of $20 \mu \mathrm{L}$. qPCR cycling conditions were as follows: initial denaturation for $2 \mathrm{~min}$ at $95.0^{\circ} \mathrm{C}$, 40 cycles of denaturation for $5 \mathrm{~s}$ at $95^{\circ} \mathrm{C}$ and annealing/extension/reading for $20 \mathrm{~s}$ at $60{ }^{\circ} \mathrm{C}$. Oligonucleotides were acquired from IDT (Integrated DNA Technologies Inc, Coralville, IA, USA) and reactions were performed on the MiniOpticonTM Real-Time PCR System (Bio-Rad, Hercules, CA, USA). The primers used were specific for Bifidobacterium longum subsp. infantis (Blon0915), total bifidobacteria (Bif) and total bacteria (Total) [33,34] (Table 1). 
Table 1. Primers used in this study.

\begin{tabular}{|c|c|c|c|}
\hline Target Microorganism & Primer Set/Probe Name & Sequence $\left(5^{\prime}-3^{\prime}\right)$ & References \\
\hline \multirow{3}{*}{ Total bacteria } & Forward (Total F) & TCCTACGGGAGGCAGCAGT & \multirow{3}{*}{ [34] } \\
\hline & Reverse (Total R) & GGACTACCAGGGTATCTAATCCTGTT & \\
\hline & Probe (Total Probe) & 5'6-FAM /CGTATTACCGCGGCTGCTGGCAC/3'TAMRA & \\
\hline \multirow{3}{*}{ genus Bifidobacterium } & Forward (Bif F) & GCGTGCTTAACACATGCAAGTC & \multirow{3}{*}{ [33] } \\
\hline & Reverse (Bif R) & CACCCGTTTCCAGGAGCTATT & \\
\hline & Probe (Bif probe) & 5'TexRd-XN/TCACGCATTACTCACCCGTTCGCC/3'IAbRQSp & \\
\hline \multirow{3}{*}{ B. longum subsp. infantis } & Forward (Blon0915-F) & CGTATTGGCTTTGTACGCATTT & \multirow{3}{*}{ [33] } \\
\hline & Reverse (Blon0915R) & ATCGTGCCGGTGAGATTTAC & \\
\hline & Probe (Blon0915 Probe) & 5'6-FAM/CCAGTATGG/ZEN/CTGGTAAAGTTCACTGCA/3'IABkFQ & \\
\hline
\end{tabular}

A standard curve was created and employed to determine the efficiency of the primers and probes by performing five 1:10 serial dilutions of B. longum subsp. infantis (DSM-20088) genomic DNA starting at $1 \mathrm{ng} / \mu \mathrm{L}$. We calculated the lower limit of detection for the multiplex assay to be $2.8 \times 10^{-6}$ ng of DNA or 1 gene-copy of $B$. longum based on the amplification data from the serial dilution and the non-template control. Because each template sample represented a different starting mass of stool, the limit of quantification for the analysis was variable from sample-to-sample, and ranged from 200 to $2 \times 10^{9}$ genecopies/g stool (B. longum subsp. infantis) and 40 to $4.5 \times 10^{9}$ gene-copies/g stool (total bifidobacteria). The relative abundance of $B$. longum subsp. infantis and total bifidobacteria was calculated as the percentage of respective taxa (gene-copies per gram stool) relative to the total bacteria (gene-copies per gram stool) quantified by qPCR. This relative abundance measure of Bifidobacterium was plotted against that of $16 \mathrm{~S}$ relative abundance to determine the extent of agreement. In calculating qPCR-based abundance, almost every DNA product amplified from target $16 \mathrm{~S}$ rDNA regions resulted in a much larger denominator and lower abundance values than $16 \mathrm{~S}$ relative abundance. Overall (Figure S2), qPCR-based relative abundance was significantly correlated with 16S-based relative abundance, although some variations across the two methods were apparent. We found that qPCR-based relative abundance was smaller than 16S-based relative abundance in the lower range of abundance values, whereas qPCR-based relative abundance was greater than 16S-based relative abundance values in the higher range of abundance. Herein, the values of gene-copies/g stool are referred to as absolute quantities to distinguish them from calculated relative abundance values.

\subsection{Statistical Analysis}

The statistical analysis was performed using $\mathrm{R}$ (version 3.5.1) [35] within RStudio (version 1.2.1335) [36]. In this study, $16 \mathrm{~S}$ microbial taxa at the phylum and genus levels, with an average relative abundance $>0.05 \%$, were subjected to downstream statistical analysis. Comparisons of categoric variables of maternal and infant characteristics were carried out using Fisher's exact tests. To determine the impact of maternal IAP and mode of delivery on infant gut microbiota at 3 months, alpha-diversity (microbial differences within samples) was calculated with four standard indices: observed OTUs, Chao1, Shannon and PD whole tree. Beta-diversity (microbial differences between samples) was analyzed by the permutational multivariate ANOVA (PERMANOVA) and a test for multivariate homogeneity of group dispersions (PERMDISP), based on Bray-Curtis dissimilarity matrices with 1000 permutations. Both analyses were conducted using QIIME2.

To determine the modifying effect of breastfeeding, statistical analyses were conducted following stratification by breastfeeding status (exclusive, partial and non-breastfeeding). The discriminative $16 \mathrm{~S}$ taxonomic biomarkers of infant gut microbiota were identified using linear discriminant analysis (LDA) effect size (LEfSe) [37], and significant differences were measured by an LDA score $>2$ and $p$ value $<0.05$, using vaginal delivery with no IAP as the reference group. The associations between Bifidobacterium and other gut microbial genera were examined using Spearman's rank correlation. False discovery rates (FDR) were 
controlled using Benjamini-Hochberg adjustment [38], with 'FSA' package in R [39]. FDRcorrected $p$-values < 0.1 were considered significantly different and displayed. Statistically significant differences in $16 \mathrm{~S}$ alpha diversity indices, relative and absolute quantity of total bifidobacteria measured by qPCR and 16S rRNA sequencing were determined using the Kruskal-Wallis test; and pairwise comparisons were conducted by Dunn's post hoc test for multiple comparison with Benjamini-Hochberg $p$ value adjustments. Independence of association between birth mode and absolute quantity of Bifidobacterium was determined by univariable and multivariable linear regressions. To improve normality of data, the absolute quantity of genus Bifidobacterium was Box-Cox transformed prior to linear regression [40]. Logistic regression modelling was utilized to assess the association between the birth mode and the colonization of B. longum subsp. Infantis.

\section{Results}

In this population cohort of 1654 full-term infants at 3 months of age (mean $=3.57$, $\mathrm{SD}=1.01), 53.75 \%$ were male. The mean gestational age was 39 weeks (mean $=39.23$, $\mathrm{SD}=1.40)$. Over half of their mothers were of Caucasian ethnicity $(74.30 \%)$, obtained a university degree $(56.83 \%)$ and did not smoke $(95.11 \%)$, and were between the ages of 30 and 39 years $(65.48 \%)$. In addition, $55.08 \%$ and $27.27 \%$ of infants were exclusively and partially breastfed, and $17.65 \%$ of infants were formula-fed. Further, 876 infants were born vaginally without maternal IAP exposure (52.96\%) and 375 were born vaginally following maternal IAP $(22.67 \%) ; 10.40 \%(n=172)$ and $13.97 \%(n=231)$ of infants were delivered by CS with and without labour, respectively. Compared with infants delivered vaginally without IAP exposure, a greater proportion of CS delivered infants, particularly those who did not experience labour, was born to mothers who were Asian, with age over 30, and overweight and obese pre-pregnancy; more infants delivered by CS with no labour were male, and partial or non-breastfed than infants delivered vaginally without IAP exposure. Meanwhile, the lowest level of postnatal infant antibiotic use was observed among vaginally delivered infants without IAP, while infants delivered by CS with labour had the highest level of postnatal antibiotic use (Table S1).

\subsection{Quantification of Bifidobacteria and B. longum subsp. infantis According to a Birth Mode}

Against a backdrop of other changes to gut microbiota, 3-month old infants with maternal IAP exposure during vaginal birth had reduced relative abundance of the phylum Actinobacteria and its the actinobacterial genus Bifidobacterium (Figure S3). Specifically, bifidobacteria were less abundant in IAP-exposed infants than those of unexposed infants who were exclusively or partially breastfed, while this difference was not significant in non-breastfed infants (Figure 1A,D). As noted, total bifidobacteria and B. longum subsp. infantis in faecal samples were further quantified by qPCR as absolute quantities in faecal samples. Maternal IAP exposure, or CS with or without labour, were associated with lower absolute quantities of genus Bifidobacterium species compared to vaginal birth with no IAP (Table S2). When comparisons were conducted within infant feeding strata in our study, statistically significant reductions in bifidobacterial quantity were observed between IAP and no IAP in vaginally delivered infants who were exclusively (6.83 vs. $7.14 \log _{10}$ (gene-copies/g faeces), $p<0.05$ ) and partially breastfed (6.75 vs. $7.25 \log _{10}$ (genecopies/g faeces), $p<0.05$, Table S2). Non-breastfed infants had lower absolute quantities of bifidobacteria at 3 months, especially if they were delivered by CS with or without labour (6.71 or $6.52 \log _{10}$ (gene-copies/g faeces) vs. $6.94 \log _{10}$ (gene-copies/g faeces) for vaginal delivery without IAP). 

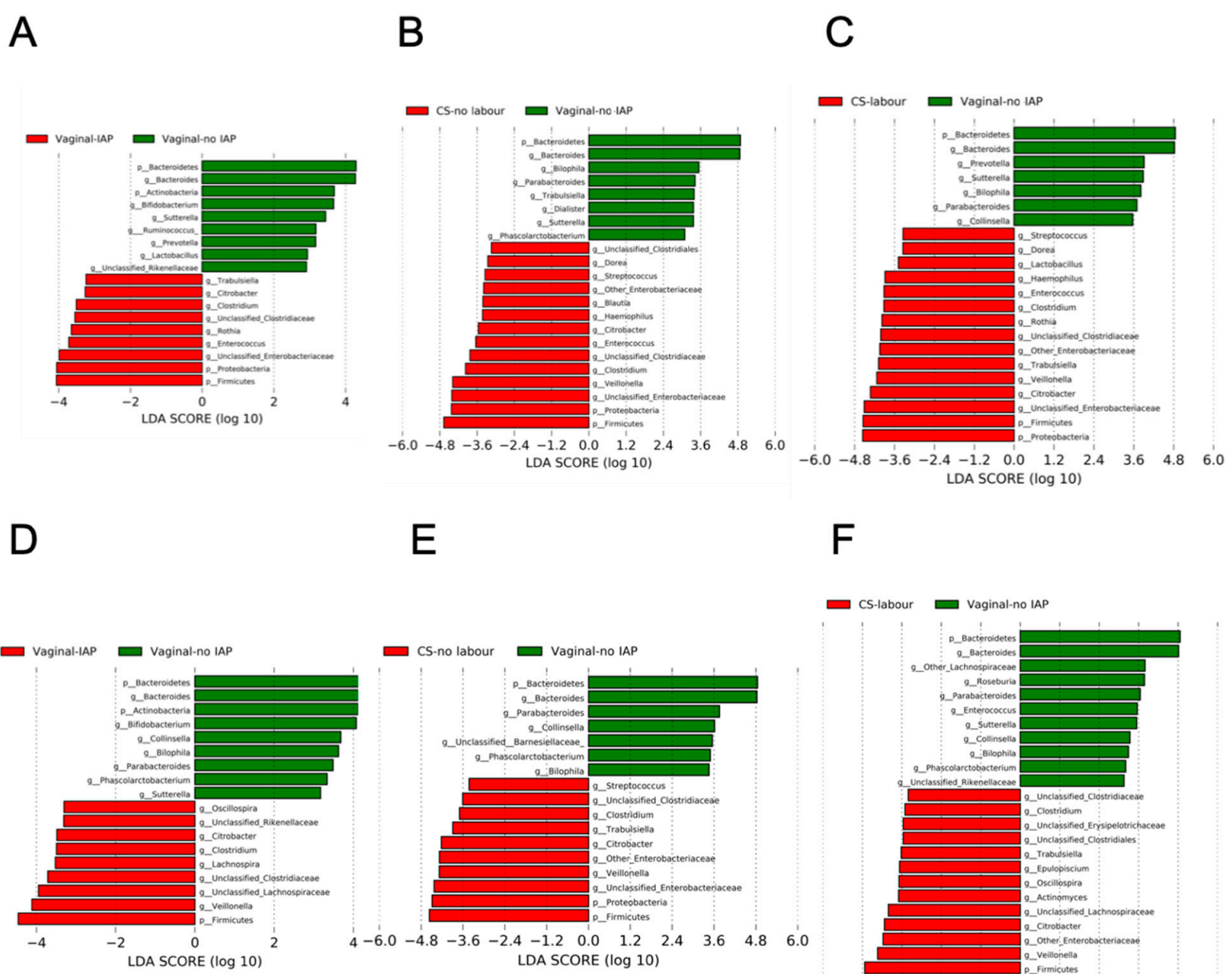

E

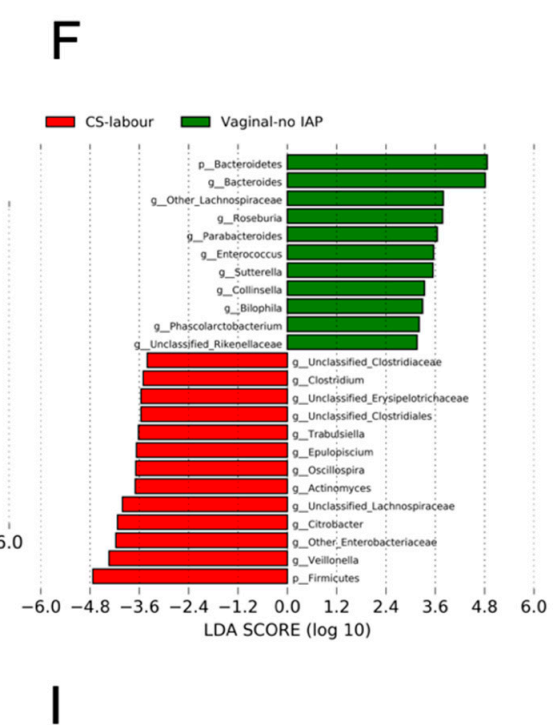

G

$\mathrm{H}$
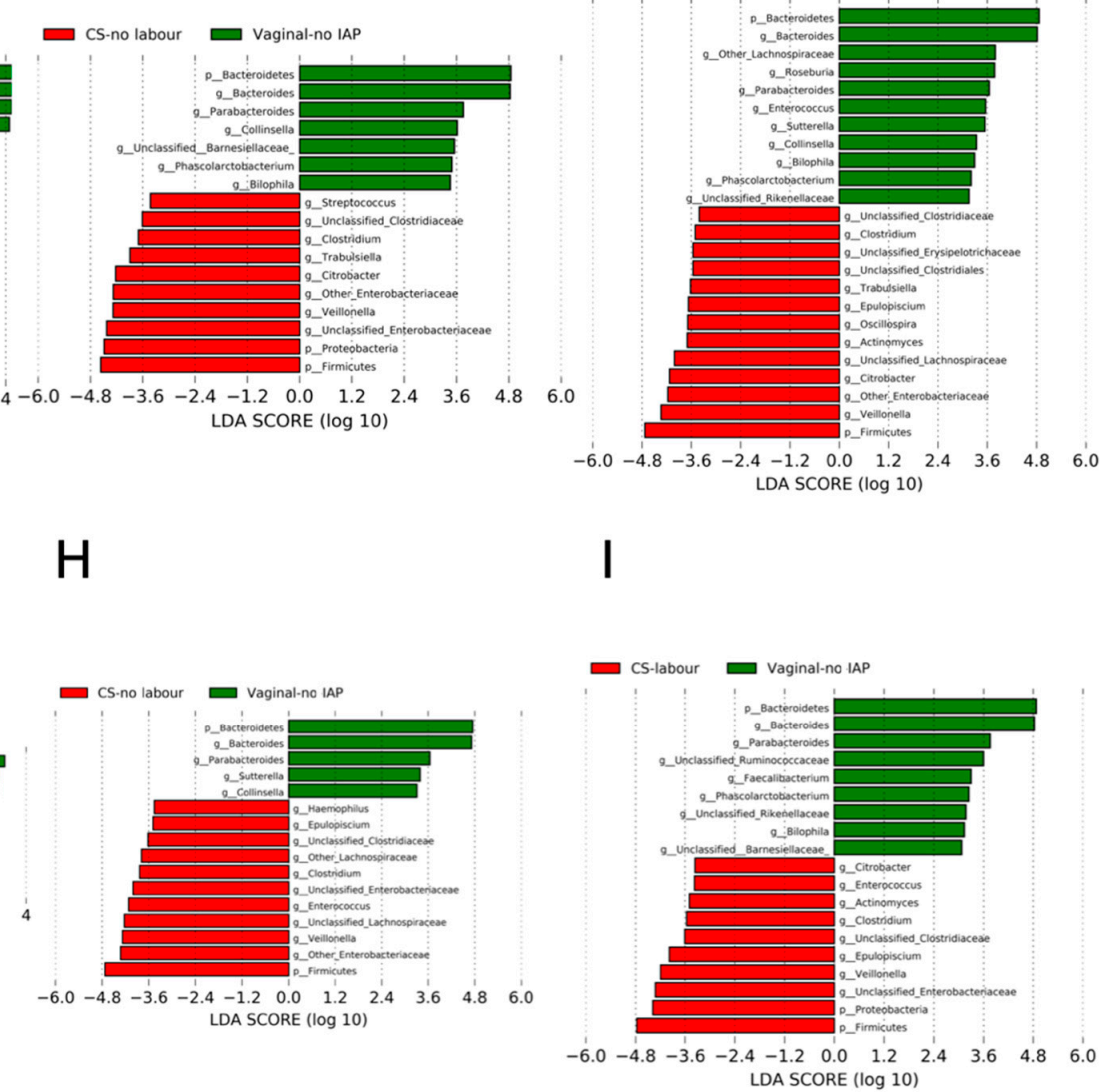

Figure 1. Linear discriminant analysis of $16 \mathrm{~S}$ gut microbiota by a birth mode within exclusively breastfed (A-C), partially breastfed (D-F) and non-breastfed (G-I) infants at 3 months.

In accordance with results from the LEfSe analysis of $16 \mathrm{~S}$ abundance data (Figure 1), vaginal delivery with maternal IAP exposure decreased the relative qPCR abundance of bifidobacteria. The median values of relative abundance were $1.50 \%$ and $1.13 \%$ among IAP-exposed infants, compared with $3.31 \%$ and $3.87 \%$ among unexposed infants who were exclusively and partially breastfed, respectively (Table S2). CS delivery, irrespective of labour presence or breastfeeding status, had no significant influence on the relative $16 \mathrm{~S}$ or qPCR abundance of bifidobacteria when compared to vaginal delivery without IAP (Figure 1B,C,E,F,H,I, 
Table S2). When testing the clinical categories of elective and emergency CS delivery, which were employed in our previous study [14], some differences in the relative abundance of qPCR bifidobacteria were found (Table S2). With exclusive breastfeeding, elective CS delivered infants had $5.29 \%$ of Bifidobacterium, while infants born by emergency CS had $1.63 \%$ of this genus in their faecal gut microbiota $(p<0.05$, measured by qPCR, Table S2). The relative abundance of qPCR bifidobacteria was also higher in elective CS vs. vaginal IAP. These differences were no longer evident when CS delivery was re-grouped as CS with and without labour (Table S2). No statistically significant differences were found when CS without labour was compared to elective CS birth, or CS with labour to emergency CS birth (Table S2).

Independent Associations between Bifidobacteria, B. longum subsp. infantis and Birth Mode

In linear regression models predicting the Box-Cox transformed absolute quantity of bifidobacteria, crude beta-coefficients were statistically significant for vaginal delivery with maternal IAP exposure ( $p<0.05$ for all infants, Table 2$)$, indicating a lower absolute quantity of gut bifidobacteria vs. the reference group of vaginal birth with no IAP. This association persisted following adjustment for maternal BMI $(p<0.05$, Table 2$)$. For comparisons conducted within infant feeding strata, statistically significant reductions in bifidobacterial quantity were observed between IAP and no IAP in vaginally delivered infants who were exclusively (crude beta-coefficient, $\mathrm{c} \beta$ : -1.01 ; adjusted beta-coefficient, $\mathrm{a} \beta$ : $-1.03, p<0.05$, Figure 2 and Table 2$)$ and partially breastfed $(c \beta$ : $-5.73, a \beta:-6.24, p<0.05$, Figure 2 and Table 2). CS delivery, regardless of labour, and emergency CS were also associated with reduced absolute quantities of bifidobacteria, independent of BMI ( $p<0.05$ for all infants, Table 2). BMI-adjusted associations were seen for CS with or without labour in non-breastfed infants $(p<0.05$, Table 2$)$. BMI adjustment revealed statistically significant reductions in absolute quantities of bifidobacteria for CS with labour or emergency CS among exclusively breastfed or non-breastfed infants $(p<0.05$, Table 2$)$.

Table 2. Associations between birth mode and the absolute quantity of genus Bifidobacterium by qPCR analysis, all and stratified by feeding status ${ }^{\dagger}$.

\begin{tabular}{|c|c|c|c|c|c|c|c|}
\hline \multicolumn{8}{|c|}{ CS Groups Defined in This Study } \\
\hline \multirow{2}{*}{$\begin{array}{l}\text { Breastfeeding } \\
\text { Status }\end{array}$} & \multirow{2}{*}{$\begin{array}{c}\text { Linear Regression } \\
\text { Model }\end{array}$} & \multicolumn{2}{|l|}{ Vaginal-IAP } & \multicolumn{2}{|c|}{ CS-No Labour } & \multicolumn{2}{|l|}{ CS-Labour } \\
\hline & & $\begin{array}{l}\text { Beta-Coefficient } \\
\text { (95\% CI) }\end{array}$ & $p$ Value & $\begin{array}{l}\text { Beta-Coefficient } \\
(95 \% \text { CI })\end{array}$ & $p$ Value & $\begin{array}{l}\text { Beta-Coefficient } \\
\text { (95\% CI) }\end{array}$ & $p$ Value \\
\hline \multirow[t]{2}{*}{ All } & Crude & $-1.12(-1.75,-0.49)$ & 0.0005 & $-0.98(-1.78,-0.19)$ & 0.02 & $-0.88(-1.75,-0.01)$ & 0.047 \\
\hline & Adjusted $\ddagger$ & $-1.18(-1.83,-0.52)$ & 0.0004 & $-1.05(-1.87,-0.22)$ & 0.01 & $-1.29(-2.19,-0.39)$ & 0.005 \\
\hline \multirow[t]{2}{*}{ Exclusive } & Crude & $-1.01(-1.88,-0.13)$ & 0.02 & $-0.66(-1.89,0.57)$ & 0.29 & $-1.03(-2.27,0.21)$ & 0.10 \\
\hline & Adjusted & $-1.03(-1.93,-0.13)$ & 0.03 & $-0.91(-2.19,0.38)$ & 0.17 & $-1.31(-2.58,-0.04)$ & 0.04 \\
\hline \multirow[t]{2}{*}{ Partial } & Crude & $-5.73(-10.00,-1.46)$ & 0.01 & $-3.96(-8.79,0.87)$ & 0.11 & $-2.09(-7.74,3.55)$ & 0.47 \\
\hline & Adjusted & $-6.24(-10.72,-1.77)$ & 0.01 & $-3.94(-8.91,1.03)$ & 0.12 & $-2.86(-8.66,2.94)$ & 0.33 \\
\hline \multirow[t]{2}{*}{ Non } & Crude & $-0.86(-2.26,0.55)$ & 0.23 & $-1.77(-3.40,-0.15)$ & 0.03 & $-1.02(-2.95,0.91)$ & 0.30 \\
\hline & Adjusted & $-0.91(-2.32,0.50)$ & 0.20 & $-1.76(-3.43,-0.09)$ & 0.04 & $-2.59(-4.61,-0.56)$ & 0.01 \\
\hline \multicolumn{8}{|c|}{ CS Groups Defined Clinically } \\
\hline \multirow{2}{*}{$\begin{array}{l}\text { Breastfeeding } \\
\text { Status }\end{array}$} & \multirow{2}{*}{$\begin{array}{c}\text { Linear Regression } \\
\text { Model }\end{array}$} & \multicolumn{2}{|l|}{ Vaginal-IAP } & \multicolumn{2}{|l|}{ CS-Elective } & \multicolumn{2}{|c|}{ CS-Emergency } \\
\hline & & $\begin{array}{l}\text { Beta-Coefficient } \\
(95 \% \mathrm{CI})\end{array}$ & $p$ Value & $\begin{array}{l}\text { Beta-Coefficient } \\
(95 \% \mathrm{CI})\end{array}$ & $p$ Value & $\begin{array}{l}\text { Beta-Coefficient } \\
(95 \% \mathrm{CI})\end{array}$ & $p$ Value \\
\hline \multirow[t]{2}{*}{ All } & Crude & $-1.12(-1.75,-0.49)$ & 0.0005 & $-0.79(-1.71,0.13)$ & 0.09 & $-1.04(-1.80,-0.27)$ & 0.01 \\
\hline & Adjusted & $-1.18(-1.83,-0.52)$ & 0.0004 & $-0.88(-1.84,0.08)$ & 0.07 & $-1.33(-2.13,-0.54)$ & 0.001 \\
\hline \multirow[t]{2}{*}{ Exclusive } & Crude & $-1.01(-1.88,-0.13)$ & 0.02 & $0.18(-1.30,1.65)$ & 0.82 & $-1.34(-2.43,-0.25)$ & 0.02 \\
\hline & Adjusted & $-1.03(-1.93,-0.13)$ & 0.03 & $-0.22(-1.78,1.33)$ & 0.78 & $-1.52(-2.65,-0.40)$ & 0.01 \\
\hline \multirow[t]{2}{*}{ Partial } & Crude & $-5.73(-9.99,-1.47)$ & 0.01 & $-5.74(-11.01,-0.47)$ & 0.03 & $-0.92(-5.98,4.14)$ & 0.72 \\
\hline & Adjusted & $-6.24(-10.71,-1.77)$ & 0.01 & $-5.72(-11.17,-0.27)$ & 0.04 & $-1.54(-6.73,3.66)$ & 0.56 \\
\hline \multirow[t]{2}{*}{ Non } & Crude & $-0.86(-2.26,0.55)$ & 0.23 & $-1.45(-3.31,0.42)$ & 0.13 & $-1.50(-3.16,0.17)$ & 0.08 \\
\hline & Adjusted & $-0.91(-2.32,0.49)$ & 0.20 & $-1.25(-3.17,0.68)$ & 0.20 & $-2.71(-4.42,-0.99)$ & 0.002 \\
\hline
\end{tabular}

${ }^{+}$Analysis is conducted by univariable and multivariable linear regression. To improve the normality, the absolute quantity of genus Bifidobacterium is Box-Cox transformed. Vaginal-no IAP is the reference group. Significant $p$ values are shown in bold. $\ddagger$ Model is adjusted for maternal BMI. 


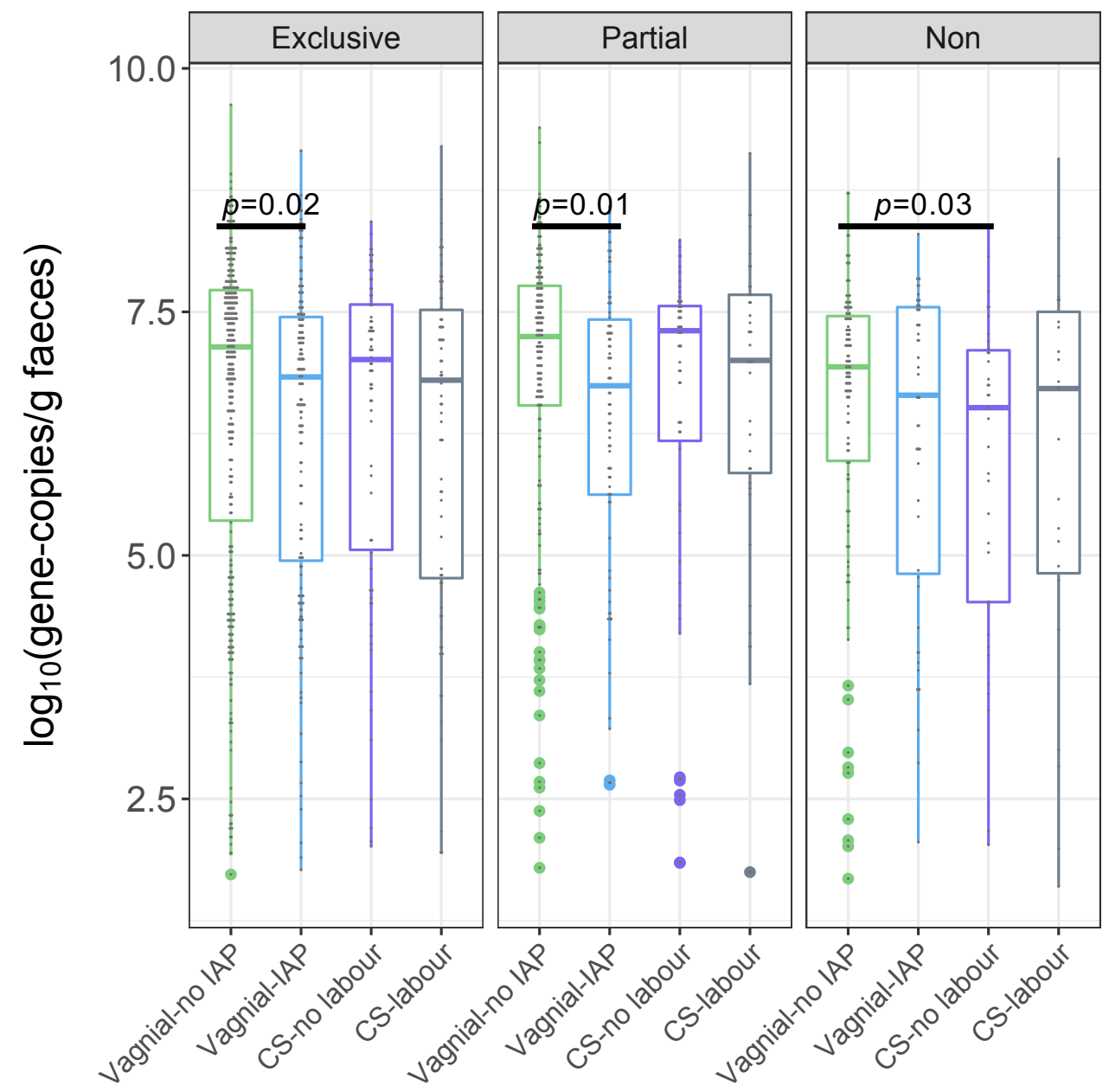

Figure 2. Birth mode comparisons of the absolute quantity of genus Bifidobacterium by qPCR within exclusively breastfed, partially breastfed and non-breastfed infants at 3 months of age. $p$ values are from crude linear regression models of the Box-Cox transformed absolute quantity of genus Bifidobacterium. Vaginal-no IAP is the reference group.

B. longum subsp. infantis was detected in only $7.30 \%$ of infants. Crude odds ratios indicated no significant association between birth mode and the colonization of $B$. longum subsp. infantis, when stratified by feeding status (Figure 3). In addition, no associations were observed following adjustment for maternal BMI. Almost attaining statistical significance was the crude OR of 0.31 (95\% CI: 0.05-1.04) for B. longum subsp. infantis following CS without labour in exclusively breastfed infants. BMI adjustment nullified this association as: OR: 0.35; 95\% CI: 0.06-1.18. 


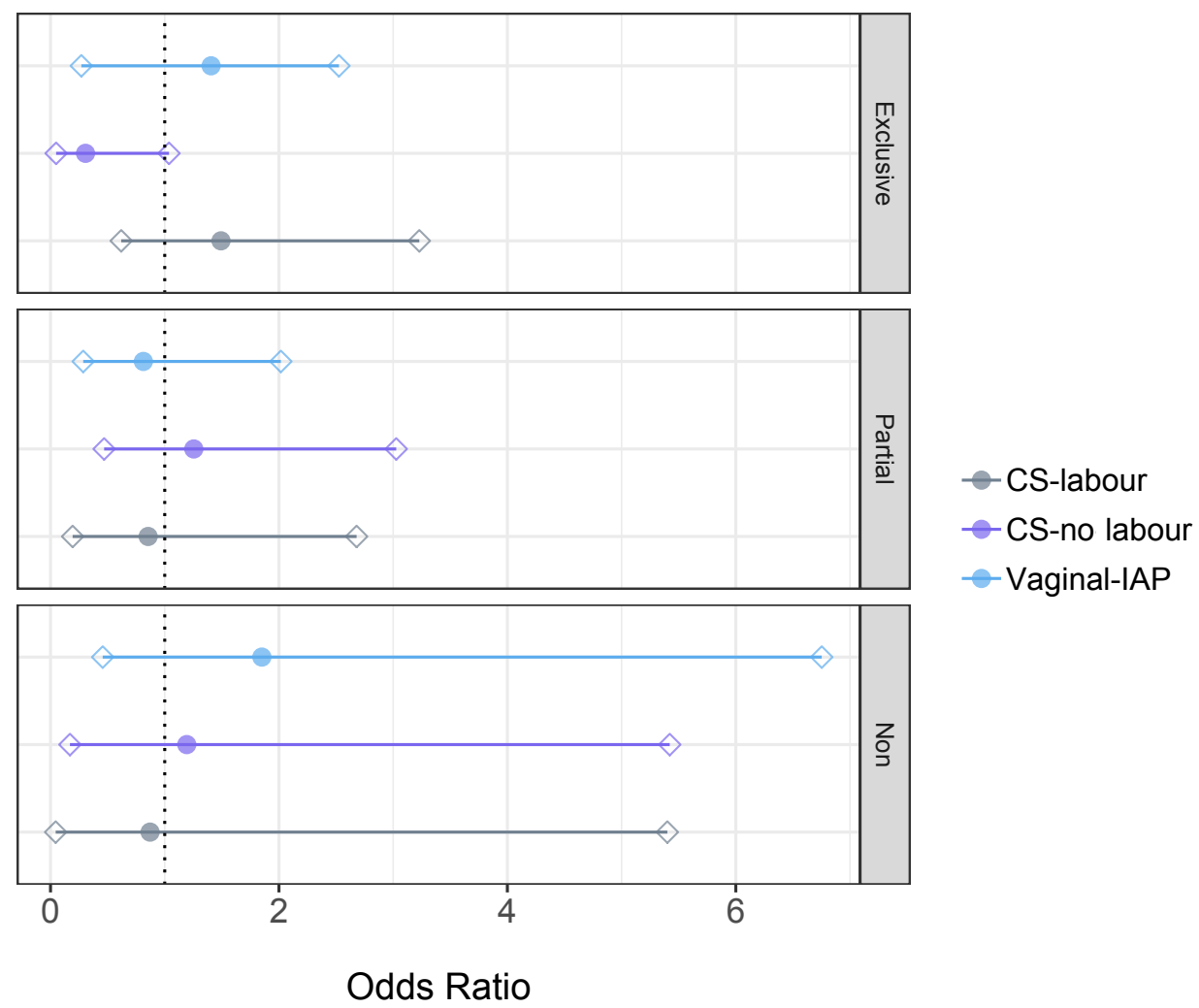

Figure 3. Likelihood (OR, 95\% CI) of gut microbial colonization with qPCR Bifidobacterium longum subsp. infantis according to birth mode within exclusively breastfed, partially breastfed and nonbreastfed infants at 3 months of age. Crude ORs from logistic regression are presented. Vaginal-no IAP is the reference group. The circle indicates the odds ratio and rhombuses indicate $95 \%$ CIs. The dotted line shows an odds ratio of 1 .

\subsection{Alpha-Diversity, Beta-Diversity of Gut Microbial Community According to a Birth Mode}

Principal Coordinates Analysis (PCoA) displayed the impact of maternal IAP on the structure of infant gut microbial communities $(p<0.05$ according to PERMANOVA and PERMDISP; Figure S4B). In addition, alpha-diversity analyses indicated that maternal IAP exposure significantly decreased the microbial richness (observed OTUs and Chao1) $(p<0.001)$ (Figure S4A). Vaginally delivered infants exposed to maternal IAP had reduced microbial diversity (Shannon and PD whole tree) compared to unexposed infants $(p \leq 0.05)$ (Figure S4A).

\subsection{Overall Gut Microbiota According to a Birth Mode, Stratified by Breastfeeding Status}

At the phylum level, Bacteroidetes was under-represented in all IAP-exposed infants, with the most severe depletion observed in CS-delivered infants. Specifically, the average relative abundance of Bacteroidetes was 38.37\% among unexposed infants, whereas it was $30.81 \%$ among vaginally born IAP-exposed infants, and less than $13.0 \%$ in CS-delivered infants (Figure S3A). In all infant feeding modes, CS with or without labour was associated with a lower abundance of Bacteroidetes and members of the genus Bacteroides than vaginal delivery with no IAP (Figure 1B,C,E,F,H,I). However, reduced abundance of Bacteroidetes and Bacteroides was solely observed following vaginal delivery with IAP in exclusively and partially breastfed infants (Figure 1A).

Maternal IAP exposure, particularly CS delivery, was associated with highly abundant Firmicutes and Proteobacteria, regardless of breastfeeding status (Figure 1 and S3). Members of genera belonging to the Firmicutes, including Enterococcus, "Clostridium", unclassified Clostridiaceae, Veillonella, were highly abundant in infants born by CS (Figure S5B,C). Members of the phylum Proteobacteria, such as unclassified Enterobacteriaceae, other En- 
terobacteriaceae, and Citrobacter, were most commonly found among CS-delivered infants whether they experienced labour or not (Figure S5B,C). With any breastfeeding, genus Citrobacter were more abundant in infant gut microbiota following IAP exposure in vaginal or CS birth (Figure 1A-F); if breastfeeding was exclusive, genus Enterococcus also became more abundant when compared to vaginal birth with no IAP (Figure 1A-C).

\subsection{Co-Occurrence Network between Bifidobacterium and Other Gut Microbiota}

Significant correlations (FDR < 0.1) between Bifidobacterium and other microbial taxa at the genus level among exclusively breastfed infants were identified and shown in Figure 4 (partially breastfed, Figure S6; non-breastfed, Figure S7). The genus Bifidobacterium had increased Shannon diversity and exhibited a strongly positive correlation with the genera Rothia, Enterococcus, Lactobacillus and Streptococcus, and a negative correlation with "Clostridium" and unclassified Clostridiaceae among exclusively breastfed infants following most all types of delivery mode, except CS delivery without labour (Figure 4). Bacteroides showed a strongly negative correlation with Bifidobacterium among vaginally delivered infants, especially those without IAP exposure (Figure 4). Members of the Proteobacteria, including species of unclassified and other Enterobacteriaceae, were negatively correlated with Bifidobacterium in infants delivered vaginally with IAP and by CS with labour (Figure 4).

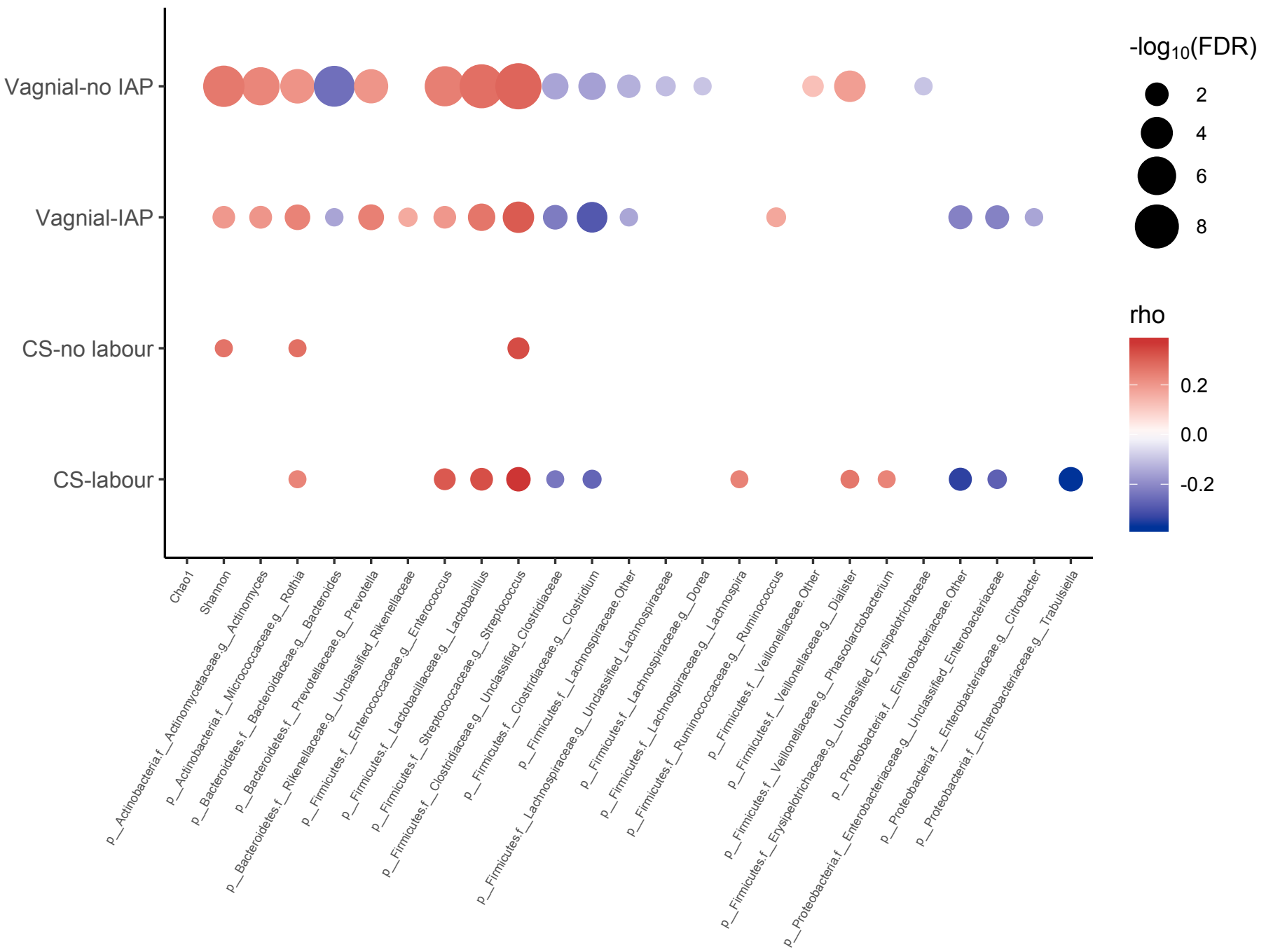

Figure 4. Correlation networks showing associations between Bifidobacterium and other gut microbial genera according to the birth mode in exclusively breastfed infants. Circle sizes and colour intensity (Red = positive, blue $=$ negative) are proportional to the significance and strength of association, respectively. Only significant (FDR $<0.1)$ correlations were displayed. 


\section{Discussion}

In a general population birth cohort of 1654 infants, maternal IAP exposure was associated with reduced absolute quantities of bifidobacteria among vaginally delivered infants (6.80 vs. $7.14 \log _{10}$ (gene-copies/g faeces), $p<0.05$ ), as well as their lowered abundance relative to other gut microbiota. These results are consistent with a systematic review of eight studies showing depletion of bifidobacteria in the gut microbiota of infants exposed to intrapartum antibiotics, although this difference did not persist to one month after birth in three studies [41]. They were also independent of maternal pre-pregnancy BMI, a determinant of bifidobacterial abundance in breast milk [42]. We also found depletion of bifidobacteria in caesarean birth, with or without labour, compared to vaginal delivery without IAP. This finding too, was observed in several studies until an infant age of 6 months [4], and more obviously in the early weeks after birth [3]. In CHILD Cohort Study women, GBS and caesarean prophylaxis were primarily implemented through intrapartum administration of penicillin and cefazolin, respectively [43]. All species and strains of Bifidobacterium are sensitive to penicillin antibiotics but less so to the cephalosporins [44]. In fact, each additional hour of IAP exposure, namely to penicllin, is reportedly associated with a 7\% reduction in the abundance of bifidobacteria in infant gut microbiota [13]. Since breast milk is a rich source of HMOs that act as "prebiotics" to promote the growth of bifidobacteria [45,46], the extent of breastfeeding by delivery type is a candidate explanation for IAP differences. Certainly, the gut microbiota of exclusively breastfed vaginally delivered infants contains the highest amounts of bifidobacteria soon after birth [47]. However, when comparisons were conducted within infant feeding strata in our study, statistically significant reductions in bifidobacterial quantity were solely observed between IAP and no IAP in vaginally delivered infants who were exclusively (6.83 vs. $7.14 \log _{10}$ (gene-copies/g faeces), $p<0.05$ ) or partially breastfed (6.75 vs. $7.25 \log _{10}$ (gene-copies/g faeces), $p<0.05$ ), even when adjusted for pre-pregnancy BMI. In the next paragraphs, we turn to findings on correlations between Bifidobacterium and other microbiota, as proxy measures for cooperation and competition among gut microbiota, to speculate on the meaning of the study's findings.

While of lesser importance than IAP in modifying whole gut microbial composition in the initial months after birth, breastfeeding plays a greater role in shaping gut microbiota with advancing age [7]. The co-existence Bifidobacterium species with other intestinal microbes was attributed to survival strategies adopted by bifidobacteria, including glycanharvesting, glycan-breakdown and cross-feeding behaviour [48]. Specifically, bifidobacteria are able to digest breast milk HMOs into small sugars, which are further metabolized by glycolytic microbes such as species of Streptococcus and Enterococcus [49]. This metabolic cross-feeding likely accounts for the positive correlation between Bifidobacterium and Streptococcus or Enterococcus in our study, as does their co-occurrence in 3-month human milk of CHILD study infants [50]. Similar to results reported by others [51], we observed an inverse correlation between Bifidobacterium and Bacteroides at 3 months of age, but solely in vaginally delivered infants. This was especially apparent in the absence of IAP, when Bifidobacterium and Bacteroides were both highly abundant and competing for HMOs [52]. Members of the phylum Proteobacteria were also inversely correlated with Bifidobacterium, the former of which were most enriched in exclusively breastfed infants delivered by CS with labour. Because bifidobacteria produce organic acids and generate a low $\mathrm{pH}$ environment [53], as well as producing antimicrobial polysaccharides [54], they create unfavorable environments for other gut microbiota. Altogether, vaginal IAP and caesarean section promote Proteobacterial blooms, propagating antibiotic resistance genes and giving these pathobionts a competitive advantage; in the case of IAP, the growth of Bifidobacterium is also inhibited [55]. Since gut microbiota of infants born following vaginal IAP are not depleted with Bacteroidetes species to nearly the same extent as after caesarean birth, their continued presence may further reduce bifidobacterial quantities as they compete for HMOs.

Three months after birth, we observed gut microbial communities following vaginal IAP or caesarean birth to be distinct from undisturbed vaginal birth in terms of betadiversity and lowered OTUs and species richness. Common across IAP and both CS 
types was over-representation by genera in the Clostridiaceae and Enterobacteriaceae families. Genus Veillonella was more abundant among CS-delivered infants. All of these findings were shown to be characteristic of CS birth in a recent meta-analysis published by Podlesny et al. [56]. Non-breastfed infants had lower absolute quantities of bifidobacteria at 3 months, especially if they were delivered by CS with or without labour. CS with labour differences were also statistically significant in exclusively breastfed infants; this is putatively a function of reduced bifidobacterial abundance with prolonged labour [10]. Mitchell, et al. [1] and Shao, et al. [7] noted a similar gut microbial composition in 2-3 week old infants born by post-labour or pre-labour CS, including one with depleted Bacteroides, but no appreciable changes to the relative abundance of Bifidobacterium. In our study, neither the absolute nor the relative Bifidobacterium quantity differed among the CS definition types: CS without labour vs. elective CS or CS with labour vs. emergency CS. However, relative abundance of Bifidobacterium was higher following elective CS compared to emergency CS delivery. Beta-diversity differences in infant gut microbiota between these clinically defined CS births were also found [57]. In fact, relative to vaginal birth with no IAP, we noted the reduction in bifidobacteria to be more robust for emergency CS than for CS with labour. When cases of pre-labour emergency CS were re-classified as CS without labour, Bifidobacterium differences were no longer evident between CS with or without labour. Prenatal complications, such as preeclampsia, a common indication for pre-labour emergency CS, may lead to reduced bifidobacteria in maternal gut microbiota of the third trimester [58] and to the transfer of bifidobacteria to the newborn.

There are several strengths of this study. Firstly, the CHILD cohort is a well-characterized birth cohort, such that we were able to investigate in-depth the impact of IAP and birth mode on infant gut microbiota. Secondly, an adequate sample size allowed us to adjust for important confounding factors, particularly breastfeeding. Last but not least, the qPCR-based abundance data were complementary to the 16S-based relative abundance data typically analysed in many microbiome studies. Although our Illumina MiSeq method was costeffective for the sequencing of the $16 \mathrm{~S}$ rRNA gene in a large number of faecal samples, several studies noted the under-representation of the genus Bifidobacterium when a universal primer set targeting the V4 region was employed. This study also used genus- and species-specific primer sets to quantify faecal Bifidobacterium and B. longum subsp. infantis to improve detection of the bifidobacteria. One limitation of this study was that we assayed only one species of Bifidobacterium (i.e., B. longum subsp. infantis) using the species-specific qPCR.

\section{Conclusions}

In conclusion, this study revealed the impact of the birth mode on the bifidobacterial abundance in infant gut microbiota after 3 months. Specifically, we found that maternal IAP exposure during vaginal birth significantly decreased the absolute and relative abundance of Bifidobacterium, even among breastfed infants. Moreover, absolute quantities of bifidobacteria were negatively affected by CS birth, with or without labour. Our epidemiological study points to the important ecological role of the genus Bifidobacterium in terms of its interaction with other gut microbiota during early infancy.

Supplementary Materials: The following are available online at https: / www.mdpi.com/article / 10.3390/microorganisms9091847/s1, Figure S1: Directed acyclic graph (DAG) of the association between the birth mode and infant gut microbiota at three months of age, Figure S2: Scatter plots with the linear regression line demonstrating the relationship between qPCR- and 16S-based relative abundance (A); and the relationship between qPCR-based absolute abundance and 16S-based relative abundance (B) for Bifidobacterium in infants aged three months, Figure S3: Relative abundance of dominant phyla (A) and genera (B) in infant gut microbiota at 3 months, according to the birth mode, Figure S4: Alpha-diversity (A) and beta-diversity (B) of gut microbial community in infants at 3 months in relation to the birth mode, Figure S5: Linear discriminant analysis of gut microbiota according to birth mode, comparing vaginal-IAP (A), CS-no labour (B), CS-labour (C), CS-elective (D), and CS-emergency (E) vs. vaginal-no IAP at 3 months, Figure S6: Correlation networks showing associations between Bifidobacterium and other gut microbial genera according to the birth mode in 
partially breastfed infants, Figure S7: Correlation networks showing associations between Bifidobacterium and other gut microbial genera according to the birth mode in non-breastfed infants, Table S1: Maternal and infant characteristics stratified by birth mode, Table S2: Median relative abundance of genus Bifidobacterium in faecal microbiota of infants according to a birth mode at 3 months by $16 \mathrm{~S}$ rRNA sequencing and qPCR analysis.

Author Contributions: Conceptualization, Y.Y.C. and A.L.K.; methodology, Y.Y.C. and A.L.K.; validation, Y.Y.C., X.Z. and A.L.K.; formal analysis, Y.Y.C. and X.Z.; resources, E.S., P.J.M., T.J.M., S.E.T., P.S., J.A.S., A.L.K.; data curation, Y.Y.C.; writing-original draft preparation, Y.Y.C.; writing-review and editing, Y.Y.C., X.Z., W.M., H.M.T., E.S., P.J.M., T.J.M., S.E.T., P.S., J.A.S., A.L.K.; supervision, A.L.K.; funding acquisition, A.L.K. All authors have read and agreed to the published version of the manuscript.

Funding: The Canadian Institutes of Health Research (CIHR) Microbiome Initiative Emerging Team Grant (no. 108,028) and CIHR Operating Grant (no. 142,432) funded this research.

Institutional Review Board Statement: The study has been approved by the Human Research Ethics Boards of the University of Alberta (Pro00010073; 1 November 2020).

Informed Consent Statement: Informed consent was obtained from all subjects involved in the study.

Data Availability Statement: Restrictions apply to the availability of these data. Data was obtained from the CHILD Cohort Study and are available via childcohort.ca (accessed on 1 May 2021) with the permission of Anita Kozyrskyj and the Child Cohort Study National Coordinating Centre.

Acknowledgments: We thank the CHILD Cohort Study (CHILD) partici pant families for their dedication and commitment to advancing health research. CHILD was initially funded by CIHR and AllerGen NCE. Visit CHILD at childcohort.ca. accessed on 1 May 2021.

Conflicts of Interest: The authors declare no conflict of interest.

\section{References}

1. Mitchell, C.M.; Mazzoni, C.; Hogstrom, L.; Bryant, A.; Bergerat, A.; Cher, A.; Pochan, S.; Herman, P.; Carrigan, M.; Sharp, K.; et al. Delivery mode affects stability of early infant gut microbiota. Cell Rep. Med. 2020, 1, 100156. [CrossRef]

2. Shaterian, N.; Abdi, F.; Ghavidel, N.; Alidost, F. Role of cesarean section in the development of neonatal gut microbiota: A systematic review. Open Med. 2021, 16, 624-639. [CrossRef]

3. Wampach, L.; Heintz-Buschart, A.; Hogan, A.; Muller, E.E.L.; Narayanasamy, S.; Laczny, C.C.; Hugerth, L.W.; Bindl, L.; Bottu, J.; Andersson, A.F.; et al. Colonization and succession within the human gut microbiome by archaea, bacteria, and microeukaryotes during the first year of life. Front. Microbiol. 2017, 8, 738. [CrossRef] [PubMed]

4. Princisval, L.; Rebelo, F.; Williams, B.L.; Coimbra, A.C.; Crovesy, L.; Ferreira, A.L.; Kac, G. Association between the mode of delivery and infant gut microbiota composition up to 6 months of age: A Systematic literature review considering the role of breastfeeding. Nutr. Rev. 2021, 1-15. [CrossRef]

5. Turroni, F.; Milani, C.; Duranti, S.; Mahony, J.; van Sinderen, D.; Ventura, M. Glycan utilization and cross-feeding activities by bifidobacteria. Trends Microbiol. 2018, 26, 339-350. [CrossRef] [PubMed]

6. Dunn, A.B.; Jordan, S.; Baker, B.J.; Carlson, N.S. The maternal infant microbiome: Considerations for labor and birth. MCN Am. J. Matern. Child. Nurs. 2017, 42, 318-325. [CrossRef]

7. Shao, Y.; Forster, S.C.; Tsaliki, E.; Vervier, K.; Strang, A.; Simpson, N.; Kumar, N.; Stares, M.D.; Rodger, A.; Brocklehurst, P.; et al. Stunted microbiota and opportunistic pathogen colonization in caesarean-section birth. Nature 2019, 574, 117-121. [CrossRef] [PubMed]

8. Mueller, N.T.; Shin, H.; Pizoni, A.; Werlang, I.C.; Matte, U.; Goldani, M.Z.; Goldani, H.A.S.; Dominguez-Bello, M.G. Delivery mode and the transition of pioneering gut-microbiota structure, composition and predicted metabolic function. Genes 2017, 8, 364 . [CrossRef]

9. Wong, W.S.W.; Sabu, P.; Deopujari, V.; Levy, S.; Shah, A.A.; Clemency, N.; Provenzano, M.; Saadoon, R.; Munagala, A.; Baker, R.; et al. Prenatal and peripartum exposure to antibiotics and cesarean section delivery are associated with differences in diversity and composition of the infant meconium microbiome. Microorganisms 2020, 8, 179. [CrossRef] [PubMed]

10. Vu, K.; Lou, W.; Tun, H.M.; Konya, T.B.; Morales-Lizcano, N.; Chari, R.S.; Field, C.J.; Guttman, D.S.; Mandal, R.; Wishart, D.S.; et al. From birth to overweight and atopic disease: Multiple and common pathways of the infant gut microbiome. Gastroenterology 2021, 160, 128-144.e110. [CrossRef]

11. Ferretti, P.; Pasolli, E.; Tett, A.; Asnicar, F.; Gorfer, V.; Fedi, S.; Armanini, F.; Truong, D.T.; Manara, S.; Zolfo, M.; et al. Mother-toinfant microbial transmission from different body sites shapes the developing infant gut microbiome. Cell Host Microbe 2018, 24, 133-145.e135. [CrossRef] 
12. Van Schalkwyk, J.; Van Eyk, N. Antibiotic prophylaxis in obstetric procedures. J. Obstet. Gynaecol. Can. 2010, 32, 878-884. [CrossRef]

13. Stearns, J.C.; Simioni, J.; Gunn, E.; McDonald, H.; Holloway, A.C.; Thabane, L.; Mousseau, A.; Schertzer, J.D.; Ratcliffe, E.M.; Rossi, L.; et al. Intrapartum antibiotics for GBS prophylaxis alter colonization patterns in the early infant gut microbiome of low risk infants. Sci. Rep. 2017, 7, 16527. [CrossRef]

14. Azad, M.B.; Konya, T.; Persaud, R.R.; Guttman, D.S.; Chari, R.S.; Field, C.J.; Sears, M.R.; Mandhane, P.J.; Turvey, S.E.; Subbarao, P.; et al. Impact of maternal intrapartum antibiotics, method of birth and breastfeeding on gut microbiota during the first year of life: A prospective cohort study. BJOG 2016, 123, 983-993. [CrossRef]

15. Alcon-Giner, C.; Caim, S.; Mitra, S.; Ketskemety, J.; Wegmann, U.; Wain, J.; Belteki, G.; Clarke, P.; Hall, L.J. Optimisation of 16S rRNA gut microbiota profiling of extremely low birth weight infants. BMC Genomics 2017, 18, 841. [CrossRef] [PubMed]

16. Walker, A.W.; Martin, J.C.; Scott, P.; Parkhill, J.; Flint, H.J.; Scott, K.P. $16 S$ rRNA gene-based profiling of the human infant gut microbiota is strongly influenced by sample processing and PCR primer choice. Microbiome 2015, 3, 26. [CrossRef]

17. O'Callaghan, A.; van Sinderen, D. Bifidobacteria and their role as members of the human gut microbiota. Front. Microbiol. 2016, 7, 925. [CrossRef]

18. Le Doare, K.; Holder, B.; Bassett, A.; Pannaraj, P.S. Mother's milk: A purposeful contribution to the development of the infant microbiota and immunity. Front. Immunol. 2018, 9, 361. [CrossRef] [PubMed]

19. Ma, J.; Li, Z.; Zhang, W.; Zhang, C.; Zhang, Y.; Mei, H.; Zhuo, N.; Wang, H.; Wang, L.; Wu, D. Comparison of gut microbiota in exclusively breast-fed and formula-fed babies: A study of 91 term infants. Sci. Rep. 2020, 10, 15792. [CrossRef] [PubMed]

20. Smilowitz, J.T.; Lebrilla, C.B.; Mills, D.A.; German, J.B.; Freeman, S.L. Breast milk oligosaccharides: Structure-function relationships in the neonate. Annu. Rev. Nutr. 2014, 34, 143-169. [CrossRef] [PubMed]

21. Forbes, J.D.; Azad, M.B.; Vehling, L.; Tun, H.M.; Konya, T.B.; Guttman, D.S.; Field, C.J.; Lefebvre, D.; Sears, M.R.; Becker, A.B.; et al. Association of exposure to formula in the hospital and subsequent infant feeding practices with gut microbiota and risk of overweight in the first year of life. JAMA Pediatr. 2018, 172, e181161. [CrossRef]

22. Ly, N.P.; Litonjua, A.; Gold, D.R.; Celedón, J.C. Gut microbiota, probiotics, and vitamin D: Interrelated exposures influencing allergy, asthma, and obesity? J. Allergy Clin. Immunol. 2011, 127, 1087-1094, quiz 1095-1086. [CrossRef]

23. Roberts, C.L.; Bell, J.C.; Ford, J.B.; Morris, J.M. Monitoring the quality of maternity care: How well are labour and delivery events reported in population health data? Paediatr. Perinat. Epidemiol. 2009, 23, 144-152. [CrossRef]

24. Tun, H.M.; Bridgman, S.L.; Chari, R.; Field, C.J.; Guttman, D.S.; Becker, A.B.; Mandhane, P.J.; Turvey, S.E.; Subbarao, P.; Sears, M.R.; et al. Roles of birth mode and infant gut microbiota in intergenerational transmission of overweight and obesity from mother to offspring. JAMA Pediatr. 2018, 172, 368-377. [CrossRef]

25. Shrier, I.; Platt, R.W. Reducing bias through directed acyclic graphs. BMC Med. Res. Methodol. 2008, 8, 70. [CrossRef]

26. Evans, D.; Chaix, B.; Lobbedez, T.; Verger, C.; Flahault, A. Combining directed acyclic graphs and the change-in-estimate procedure as a novel approach to adjustment-variable selection in epidemiology. BMC Med. Res. Methodol. 2012, 12, 156. [CrossRef] [PubMed]

27. Van der Leek, A.P.; Bahreinian, S.; Chartier, M.; Dahl, M.E.; Azad, M.B.; Brownell, M.D.; Kozyrskyj, A.L. Maternal distress during pregnancy and recurrence in early childhood predicts atopic dermatitis and asthma in childhood. Chest 2020, 158, 57-67. [CrossRef] [PubMed]

28. Azad, M.B.; Konya, T.; Guttman, D.S.; Field, C.J.; Sears, M.R.; HayGlass, K.T.; Mandhane, P.J.; Turvey, S.E.; Subbarao, P.; Becker, A.B.; et al. Infant gut microbiota and food sensitization: Associations in the first year of life. Clin. Exp. Allergy 2015, 45, 632-643. [CrossRef] [PubMed]

29. Caporaso, J.G.; Kuczynski, J.; Stombaugh, J.; Bittinger, K.; Bushman, F.D.; Costello, E.K.; Fierer, N.; Peña, A.G.; Goodrich, J.K.; Gordon, J.I.; et al. QIIME allows analysis of high-throughput community sequencing data. Nat. Methods 2010, 7, 335-336. [CrossRef]

30. DeSantis, T.Z.; Hugenholtz, P.; Larsen, N.; Rojas, M.; Brodie, E.L.; Keller, K.; Huber, T.; Dalevi, D.; Hu, P.; Andersen, G.L. Greengenes, a chimera-checked $16 \mathrm{~S}$ rRNA gene database and workbench compatible with ARB. Appl. Environ. Microbiol. 2006, 72, 5069-5072. [CrossRef]

31. Wang, Q.; Garrity, G.M.; Tiedje, J.M.; Cole, J.R. Naïve Bayesian classifier for rapid assignment of rRNA sequences into the new bacterial taxonomy. Appl. Environ. Microbiol. 2007, 73, 5261-5267. [CrossRef] [PubMed]

32. Drall, K.M.; Tun, H.M.; Morales-Lizcano, N.P.; Konya, T.B.; Guttman, D.S.; Field, C.J.; Mandal, R.; Wishart, D.S.; Becker, A.B.; Azad, M.B.; et al. Clostridioides difficile colonization is differentially associated with gut microbiome profiles by infant feeding modality at 3-4 months of age. Front. Immunol. 2019, 10, 2866. [CrossRef]

33. Frese, S.A.; Hutton, A.A.; Contreras, L.N.; Shaw, C.A.; Palumbo, M.C.; Casaburi, G.; Xu, G.; Davis, J.C.C.; Lebrilla, C.B.; Henrick, B.M.; et al. Persistence of supplemented Bifidobacterium longum subsp. infantis EVC001 in breastfed infants. mSphere 2017, 2, e00501-17. [PubMed]

34. Nadkarni, M.A.; Martin, F.E.; Jacques, N.A.; Hunter, N. Determination of bacterial load by real-time PCR using a broad-range (universal) probe and primers set. Microbiology 2002, 148, 257-266. [CrossRef] [PubMed]

35. R Core Team. R: A Language and Environment for Statistical Computing; R Foundation for Statistical Computing: Vienna, Austria, 2020; Available online: https:/ / www.R-project.org/ (accessed on 1 May 2021). 
36. RStudio Team. RStudio: Integrated Development for R; RStudio, PBC: Boston, MA, USA, 2020; Available online: http://www. rstudio.com/ (accessed on 1 May 2021).

37. Segata, N.; Izard, J.; Waldron, L.; Gevers, D.; Miropolsky, L.; Garrett, W.S.; Huttenhower, C. Metagenomic biomarker discovery and explanation. Genome Biol. 2011, 12, R60. [CrossRef]

38. Benjamini, Y.; Hochberg, Y. Controlling the false discovery rate: A practical and powerful approach to multiple testing. J. R. Stat. Soc. Ser. B 1995, 57, 289-300. [CrossRef]

39. Ogle, D.H.; Doll, J.C.; Wheeler, P.; Dinno, A. FSA: Fisheries stock analysis. R Package Version 0.9.1.9000. 2021. Available online: https:/ / github.com/droglenc/FSA (accessed on 1 May 2021).

40. Osborne, J. Improving your data transformations: Applying the Box-Cox transformation. Pract. Assess. Res. Eval. $2010,15,12$.

41. Grech, A.; Collins, C.E.; Holmes, A.; Lal, R.; Duncanson, K.; Taylor, R.; Gordon, A. Maternal exposures and the infant gut microbiome: A systematic review with meta-analysis. Gut Microbes 2021, 13, 1-30. [CrossRef]

42. Cortés-Macías, E.; Selma-Royo, M.; Martínez-Costa, C.; Collado, M.C. Breastfeeding practices influence the breast milk microbiota depending on pre-gestational maternal BMI and weight gain over pregnancy. Nutrients 2021, 13, 1518. [CrossRef] [PubMed]

43. Persaud, R.R.; Azad, M.B.; Chari, R.S.; Sears, M.R.; Becker, A.B.; Kozyrskyj, A.L. Perinatal antibiotic exposure of neonates in Canada and associated risk factors: A population-based study. J. Matern. Fetal Neonatal. Med. 2015, 28, 1190-1195. [CrossRef] [PubMed]

44. Moubareck, C.; Gavini, F.; Vaugien, L.; Butel, M.J.; Doucet-Populaire, F. Antimicrobial susceptibility of bifidobacteria. J. Antimicrob. Chemother. 2005, 55, 38-44. [CrossRef] [PubMed]

45. Lawson, M.A.E.; O’Neill, I.J.; Kujawska, M.; Gowrinadh Javvadi, S.; Wijeyesekera, A.; Flegg, Z.; Chalklen, L.; Hall, L.J. Breast milkderived human milk oligosaccharides promote Bifidobacterium interactions within a single ecosystem. ISME J 2020, 14, 635-648. [CrossRef] [PubMed]

46. Marcobal, A.; Sonnenburg, J.L. Human milk oligosaccharide consumption by intestinal microbiota. Clin. Microbiol. Infect. 2012, 18 (Suppl. 4), 12-15. [CrossRef] [PubMed]

47. Sakwinska, O.; Foata, F.; Berger, B.; Brüssow, H.; Combremont, S.; Mercenier, A.; Dogra, S.; Soh, S.E.; Yen, J.C.K.; Heong, G.Y.S.; et al. Does the maternal vaginal microbiota play a role in seeding the microbiota of neonatal gut and nose? Benef. Microbes 2017, 8, 763-778. [CrossRef]

48. Turroni, F.; Milani, C.; Duranti, S.; Mancabelli, L.; Mangifesta, M.; Viappiani, A.; Lugli, G.A.; Ferrario, C.; Gioiosa, L.; Ferrarini, A.; et al. Deciphering bifidobacterial-mediated metabolic interactions and their impact on gut microbiota by a multi-omics approach. ISME J 2016, 10, 1656-1668. [CrossRef]

49. Chassard, C.; de Wouters, T.; Lacroix, C. Probiotics tailored to the infant: A window of opportunity. Curr. Opin. Biotechnol. 2014, 26, 141-147. [CrossRef]

50. Fehr, K.; Moossavi, S.; Sbihi, H.; Boutin, R.C.T.; Bode, L.; Robertson, B.; Yonemitsu, C.; Field, C.J.; Becker, A.B.; Mandhane, P.J.; et al. Breastmilk Feeding practices are associated with the co-occurrence of bacteria in mothers' milk and the infant gut: The CHILD cohort study. Cell Host Microbe 2020, 28, 285-297.e284. [CrossRef]

51. Wang, M.; Li, M.; Wu, S.; Lebrilla, C.B.; Chapkin, R.S.; Ivanov, I.; Donovan, S.M. Fecal microbiota composition of breast-fed infants is correlated with human milk oligosaccharides consumed. J. Pediatr. Gastroenterol. Nutr. 2015, 60, 825-833. [CrossRef]

52. Marcobal, A.; Barboza, M.; Froehlich, J.W.; Block, D.E.; German, J.B.; Lebrilla, C.B.; Mills, D.A. Consumption of human milk oligosaccharides by gut-related microbes. J. Agric. Food Chem. 2010, 58, 5334-5340. [CrossRef]

53. Fukuda, S.; Toh, H.; Hase, K.; Oshima, K.; Nakanishi, Y.; Yoshimura, K.; Tobe, T.; Clarke, J.M.; Topping, D.L.; Suzuki, T.; et al. Bifidobacteria can protect from enteropathogenic infection through production of acetate. Nature 2011, 469, 543-547. [CrossRef]

54. Fanning, S.; Hall, L.J.; Cronin, M.; Zomer, A.; MacSharry, J.; Goulding, D.; Motherway, M.O.; Shanahan, F.; Nally, K.; Dougan, G.; et al. Bifidobacterial surface-exopolysaccharide facilitates commensal-host interaction through immune modulation and pathogen protection. Proc. Natl. Acad. Sci. USA 2012, 109, 2108-2113. [CrossRef]

55. Li, X.; Stokholm, J.; Brejnrod, A.; Vestergaard, G.A.; Russel, J.; Trivedi, U.; Thorsen, J.; Gupta, S.; Hjelmsø, M.H.; Shah, S.A.; et al. The infant gut resistome associates with E. coli, environmental exposures, gut microbiome maturity, and asthma-associated bacterial composition. Cell Host Microbe 2021, 29, 975-987.e974. [CrossRef] [PubMed]

56. Podlesny, D.; Fricke, W.F. Strain inheritance and neonatal gut microbiota development: A meta-analysis. Int. J. Med. Microbiol. 2021, 311, 151483. [CrossRef] [PubMed]

57. Levin, A.M.; Sitarik, A.R.; Havstad, S.L.; Fujimura, K.E.; Wegienka, G.; Cassidy-Bushrow, A.E.; Kim, H.; Zoratti, E.M.; Lukacs, N.W.; Boushey, H.A.; et al. Joint effects of pregnancy, sociocultural, and environmental factors on early life gut microbiome structure and diversity. Sci. Rep. 2016, 6, 31775. [CrossRef] [PubMed]

58. Sun, B.M.; Meng, L.; Liu, H.; Bao, D. Changes in intestinal flora in preeclampsia rats and effects of probiotics on their inflammation and blood pressure. Eur. Rev. Med. Pharmacol. Sci. 2020, 24, 10155-10161. [PubMed] 